\title{
CONGRUENCES FOR TRACES OF SINGULAR MODULI
}

\author{
ROBERT OSBURN
}

\begin{abstract}
We extend a result of Ahlgren and Ono 1 on congruences for traces of singular moduli of level 1 to traces defined in terms of Hauptmodul associated to certain groups of genus 0 of higher levels.
\end{abstract}

\section{INTRODUCTION}

Let $j(z)$ denote the usual elliptic modular function on $\mathrm{SL}_{2}(\mathbb{Z})$ with $q$-expansion $(q:=$ $\left.e^{2 \pi i z}\right)$

$$
j(z)=q^{-1}+744+196884 q+21493760 q^{2}+\cdots .
$$

The values of $j(z)$ at imaginary quadratic arguments in the upper half of the complex plane are known as singular moduli. Singular moduli are important algebraic integers which generate ring class field extensions of imaginary quadratic fields (Theorem 11.1 in [5]), are related to supersingular elliptic curves ([1]), and to Borcherds products of modular forms $(2,3])$.

Let $d$ denote a positive integer congruent to 0 or 3 modulo 4 so that $-d$ is the discriminant of an order in an imaginary quadratic field. Denote by $\mathcal{Q}_{d}$ the set of positive definite integral binary quadratic forms

$$
Q(x, y)=a x^{2}+b x y+c y^{2}
$$

with discriminant $-d=b^{2}-4 a c$. To each $Q \in \mathcal{Q}_{d}$, let $\alpha_{Q}$ be the unique complex number in the upper half plane which is a root of $Q(x, 1)$; the singular modulus $j\left(\alpha_{Q}\right)$ depends only on the equivalence class of $Q$ under the action of $\Gamma:=P S L_{2}(\mathbb{Z})$. Define $\omega_{Q} \in\{1,2,3\}$ by

$$
\omega_{Q}:= \begin{cases}2 & \text { if } Q \sim_{\Gamma}[a, 0, a] \\ 3 & \text { if } Q \sim_{\Gamma}[a, a, a], \\ 1 & \text { otherwise }\end{cases}
$$

Let $J(z)$ be the Hauptmodul

$$
J(z):=j(z)-744=q^{-1}+196884 q+21493760 q^{2}+\cdots .
$$

Zagier [15] defined the trace of the singular moduli of discriminant $-d$ as

$$
t(d):=\sum_{Q \in \mathcal{Q}_{d} / \Gamma} \frac{J\left(\alpha_{Q}\right)}{\omega_{Q}}=\sum_{Q \in \mathcal{Q}_{d} / \Gamma} \frac{j\left(\alpha_{Q}\right)-744}{\omega_{Q}} \in \mathbb{Z} .
$$

Zagier has shown that $t(d)$ has some interesting properties. Namely, the following result (see Theorem 1 in [15]) shows that the $t(d)$ 's are Fourier coefficients of a halfintegral weight modular form.

2000 Mathematics Subject Classification. Primary 11F33, 11F37; Secondary 11F50. 
Theorem 1.1. Let $\theta_{1}(z)$ and $E_{4}(z)$ be defined by

$$
\begin{aligned}
& E_{4}(z):=1+240 \sum_{n=1}^{\infty} \frac{n^{3} q^{n}}{1-q^{n}}, \\
& \theta_{1}(z):=\frac{\eta^{2}(z)}{\eta(2 z)}=\sum_{n=-\infty}^{\infty}(-1)^{n} q^{n^{2}}=1-2 q+2 q^{4}-2 q^{9}+\cdots .
\end{aligned}
$$

and let $g(z)$ be defined by

$$
g(z):=-q^{-1}+2+\sum_{0<d \equiv 0,3(\bmod 4)} t(d) q^{d}
$$

Then

$$
\begin{aligned}
g(z) & =-\frac{\theta_{1}(z) E_{4}(4 z)}{\eta^{6}(4 z)} \\
& =-q^{-1}+2-248 q^{3}+492 q^{4}-4119 q^{7} \cdots
\end{aligned}
$$

i.e., $g(z)$ is a modular form of weight $\frac{3}{2}$ on $\Gamma_{0}(4)$, holomorphic on the upper half plane and meromorphic at the cusps.

Now what about divisibility properties of $t(d)$ as $d$ varies? In this direction, Ahlgren and Ono [1] recently proved the following result which shows that these traces $t(d)$ satisfy congruences based on the factorization of primes in certain imaginary quadratic fields.

Theorem 1.2. If $d$ is a positive integer for which an odd prime l splits in $\mathbb{Q}(\sqrt{-d})$, then

$$
t\left(l^{2} d\right) \equiv 0 \quad(\bmod l) .
$$

Recently, Kim [11] and Zagier [15] defined an analogous trace of singular moduli by replacing the $j$-function by a modular function of higher level, in particular by the Hauptmodul associated to other groups of genus 0 . Let $\Gamma_{0}(N)^{*}$ be the group generated by $\Gamma_{0}(N)$ and all Atkin-Lehner involutions $W_{e}$ for $e \| N$, i.e., $e$ is a positive divisor of $N$ for which $\operatorname{gcd}(e, N / e)=1$. There are only finitely many values of $N$ for which $\Gamma_{0}(N)^{*}$ is of genus 0 (see [8, 9], or [14]). In particular, there are only finitely many prime values of $N$. For such a prime $p$, let $j_{p}^{*}$ be the corresponding Hauptmodul. For these primes $p$, Kim and Zagier define a trace $t^{(p)}(d)$ (see Section 3 below) in terms of singular values of $j_{p}^{*}$. The goal of this paper is to prove that the same type of congruence holds for $t^{(p)}(d)$, namely

Theorem 1.3. Let $p$ be a prime for which $\Gamma_{0}(p)^{*}$ is of genus 0 . If $d$ is a positive integer such that $-d$ is congruent to a square modulo $4 p$ and for which an odd prime $l \neq p$ splits in $\mathbb{Q}(\sqrt{-d})$, then

$$
t^{(p)}\left(l^{2} d\right) \equiv 0 \bmod l .
$$

\section{Preliminaries on Modular and Jacobi forms}

We first recall some facts about half-integral weight modular forms (see [12, 13]). If $f(z)$ is a function of the upper half-plane, $\lambda \in \frac{1}{2} \mathbb{Z}$, and $\left(\begin{array}{ll}a & b \\ c & d\end{array}\right) \in G L_{2}^{+}(\mathbb{R})$, then we define the slash operator by

$$
\left.f(z)\right|_{\lambda}\left(\begin{array}{ll}
a & b \\
c & d
\end{array}\right):=(a d-b c)^{\frac{\lambda}{2}}(c z+d)^{-\lambda} f\left(\frac{a z+b}{c z+d}\right)
$$


Here we take the branch of the square root having non-negative real part. If $\gamma=$ $\left(\begin{array}{ll}a & b \\ c & d\end{array}\right) \in \Gamma_{0}(4)$, then define

where

$$
j(\gamma, z):=\left(\frac{c}{d}\right) \epsilon_{d}^{-1} \sqrt{c z+d}
$$

$$
\epsilon:= \begin{cases}1 & \text { if } d \equiv 1 \quad(\bmod 4) \\ i & \text { if } d \equiv-1 \quad(\bmod 4) .\end{cases}
$$

If $k$ is an integer and $N$ is an odd positive integer, then let $\mathcal{M}_{k+\frac{1}{2}}\left(\Gamma_{0}(4 N)\right)$ denote the infinite dimensional vector space of nearly holomorphic modular forms of weight $k+\frac{1}{2}$ on $\Gamma_{0}(4 N)$. These are functions $f(z)$ which are holomorphic on the upper half-plane, meromorphic at the cusps, and which satisfy

$$
f(\gamma z)=j(\gamma, z)^{2 k+1} f(z)
$$

for all $\gamma \in \Gamma_{0}(4 N)$. Denote by $\mathcal{M}_{k+\frac{1}{2}}^{+}\left(\Gamma_{0}(4 N)\right)$ the "Kohnen plus-spaces" (see [13]) of nearly holomorphic forms which transform according to (1) and which have a Fourier expansion of the form

$$
\sum_{(-1)^{k} n \equiv 0,1(\bmod 4)} a(n) q^{n} .
$$

We recall some properties of Hecke operators on $\mathcal{M}_{k+\frac{1}{2}}^{+}\left(\Gamma_{0}(4 N)\right)$. If $l$ is a prime such that $l \nmid N$, then the Hecke operator $T_{k+\frac{1}{2}, 4 N}\left(l^{2}\right)$ on a modular form

$$
f(z):=\sum_{(-1)^{k} n \equiv 0,1(\bmod 4)} a(n) q^{n} \in \mathcal{M}_{k+\frac{1}{2}}^{+}\left(\Gamma_{0}(4 N)\right)
$$

is given by

$$
f(z) \mid T_{k+\frac{1}{2}, 4 N}\left(l^{2}\right):=\sum_{(-1)^{k} n \equiv 0,1(\bmod 4)}\left(a\left(l^{2} n\right)+\left(\frac{(-1)^{k} n}{l}\right) l^{k-1} a(n)+l^{2 k-1} a\left(n / l^{2}\right)\right) q^{n}
$$

where $\left(\frac{*}{l}\right)$ is a Legendre symbol. Let us now recall some facts about Jacobi forms (see [6]). A Jacobi form on $\mathrm{SL}_{2}(\mathbb{Z})$ is a holomorphic function

$$
\phi: \mathfrak{H} \times \mathbb{C} \rightarrow \mathbb{C}
$$

satisfying

$$
\begin{gathered}
\phi\left(\frac{a \tau+b}{c \tau+d}, \frac{z}{c \tau+d}\right)=(c \tau+d)^{k} e^{2 \pi i N \frac{c z^{2}}{c \tau+d}} \phi(\tau, z) \\
\phi(\tau, z+\lambda \tau+\mu)=e^{-2 \pi i N\left(\lambda^{2} \tau+2 \lambda z\right)} \phi(\tau, z)
\end{gathered}
$$

for all $\left(\begin{array}{ll}a & b \\ c & d\end{array}\right) \in \mathrm{SL}_{2}(\mathbb{Z})$ and $(\lambda, \mu) \in \mathbb{Z}^{2}$, and having a Fourier expansion of the form $\left(q=e^{2 \pi i \tau}, \zeta=e^{2 \pi i z}\right)$

$$
\phi(\tau, z)=\sum_{n=0}^{\infty} \sum_{\substack{r \in \mathbb{Z} \\ r^{2} \leq 4 N n}} c(n, r) q^{n} \zeta^{r} .
$$

Here $k$ and $N$ are the weight and index of $\phi$, respectively. Let $J_{k, N}$ denote the space of Jacobi forms of weight $k$ and index $N$ on $\mathrm{SL}_{2}(\mathbb{Z})$. By Theorem 2.2 in $\underline{\underline{6}}$, the coefficient $c(n, r)$ depends only on $4 N n-r^{2}$ and $r \bmod 2 N$. By definition $c(n, r)=0$ unless $4 N n-$ 
$r^{2} \geq 0$. If we drop the condition $4 N n-r^{2} \geq 0$, we obtain a nearly holomorphic Jacobi form. Let $J_{k, N}^{!}$be the space of nearly holomorphic Jacobi forms of weight $k$ and index $N$.

\section{TRACES}

Let $\Gamma_{0}(N)^{*}$ be the group generated by $\Gamma_{0}(N)$ and all Atkin-Lehner involutions $W_{e}$ for $e \| N$, that is, $e$ is a positive divisor of $N$ for which $\operatorname{gcd}(e, N / e)=1$. $W_{e}$ can be represented by a matrix of the form $\frac{1}{\sqrt{e}}\left(\begin{array}{cc}e x & y \\ N z & e w\end{array}\right)$ with $x, y, z, w \in \mathbb{Z}$ and $x w e-y z N / e=1$. There are only finitely many values of $N$ for which $\Gamma_{0}(N)^{*}$ is of genus 0 (see [8], 9], or [14). In particular, if we let $\mathfrak{S}$ denote the set of prime values for such $N$, then

$$
\mathfrak{S}=\{2,3,5,7,11,13,17,19,23,29,31,41,47,59,71\} .
$$

For $p \in \mathfrak{S}$, let $j_{p}^{*}$ be the corresponding Hauptmodul with Fourier expansion

$$
q^{-1}+0+a_{1} q+a_{2} q^{2}+\ldots
$$

Let us now define a trace in terms of the $j_{p}^{*}$ 's. Let $d$ be a positive integer such that $-d$ is congruent to a square modulo $4 p$. Choose an integer $\beta \bmod 2 p$ such that $\beta^{2} \equiv-d \bmod 4 p$ and consider the set

$$
\mathcal{Q}_{d, p, \beta}=\left\{[a, b, c] \in \mathcal{Q}_{d}: a \equiv 0 \bmod p, b \equiv \beta \bmod 2 p\right\} .
$$

Note that $\Gamma_{0}(p)$ acts on $\mathcal{Q}_{d, p, \beta}$. Assume that $d$ is not divisible as a discriminant by the square of any prime dividing $p$, i.e. not divisible by $p^{2}$. Then we have a bijection via the natural map between

$$
\mathcal{Q}_{d, p, \beta} / \Gamma_{0}(p)
$$

and

$$
\mathcal{Q}_{d} / \Gamma
$$

as the image of the root $\alpha_{Q}, Q \in \mathcal{Q}_{d, p, \beta}$, in $\Gamma_{0}(p) / \mathfrak{H}$ corresponds to a Heegner point. We could then define a trace $t^{(p, \beta)}(d)$ as the sum of the values of $j_{p}^{*}$ with $Q$ running over a set of representatives for $\mathcal{Q}_{d, p, \beta} / \Gamma_{0}(p)$. As $t^{(p, \beta)}(d)$ is independent of $\beta$, we define the trace $t^{(p)}(d)$ (see Section 8 of [15] or Section 1 of [11])

$$
t^{(p)}(d)=\sum_{Q} \frac{j_{p}^{*}\left(\alpha_{Q}\right)}{\omega_{Q}} \in \mathbb{Z}
$$

where the sum is over $\Gamma_{0}(p)^{*}$ representatives of forms $Q=[a, b, c]$ satisfying $a \equiv 0 \bmod p$.

Remark 3.1. For $p=2$, we have $t^{(2)}(4)=\frac{1}{2} j_{2}^{*}\left(\frac{1+i}{2}\right)=-52, t^{(2)}(7)=j_{2}^{*}\left(\frac{1+\sqrt{-7}}{4}\right)=-23$, $t^{(2)}(8)=j_{2}^{*}\left(\frac{\sqrt{-2}}{2}\right)=152$. For $p=3$, we have $t^{(3)}(3)=\frac{1}{3} j_{3}^{*}\left(\frac{-3+\sqrt{-3}}{6}\right)=-14, t^{(3)}(11)=$ $j_{3}^{*}\left(\frac{1+\sqrt{-11}}{6}\right)=22$. Moreover by the table in Section 8 of [15], we have: 


\begin{tabular}{c|ccc}
$d$ & $t^{(2)}(d)$ & $t^{(3)}(d)$ & $t^{(5)}(d)$ \\
\hline 3 & \multicolumn{3}{|c}{-14} \\
\hline 4 & -52 & & -8 \\
\hline 7 & -23 & & \\
\hline 8 & 152 & -34 & \\
\hline 11 & \multicolumn{3}{|c}{22} \\
\hline 12 & -496 & 52 & -12 \\
\hline 15 & -1 & -138 & -38 \\
\hline 16 & 1036 & & -6 \\
\hline 19 & \multicolumn{3}{|c}{20} \\
\hline 20 & -2256 & -116 & 12 \\
\hline 23 & -94 & 115 & \\
\hline 24 & 4400 & 348 & -44 \\
\hline 27 & \multicolumn{3}{|c}{-482} \\
\hline 28 & -8192 & &
\end{tabular}

The empty entries correspond to $-d$ which are not congruent to squares modulo $4 p$.

By the discussion in Section 8 of [15] or Section 2.2 in [11], there exist forms $\phi_{p} \in J_{2, p}^{!}$ uniquely characterized by the condition that their Fourier coefficients $c(n, r)=B(4 p n-$ $r^{2}$ ) depend only on $r^{2}-4 p n$ and where $B(-1)=1, B(d)=0$ if $d=4 p n-r^{2}<0, \neq-1$ and $B(0)=-2$. Define $g_{p}(z)$ as

$$
g_{p}(z):=q^{-1}+\sum_{d \geq 0} B(d) q^{d} .
$$

By the correspondence between Jacobi forms and half-integral weight forms (Theorem 5.6 in [6] $), g_{p}(z) \in \mathcal{M}_{\frac{3}{2}}^{+}\left(\Gamma_{0}(4 p)\right)$. As the dimension of $J_{2, p}$ is zero, we have that for every integer $d \geq 0$ such that $-d$ is congruent to a square modulo $4 p$, there exists a unique $f_{d, p} \in \mathcal{M}_{\frac{1}{2}}^{+}\left(\Gamma_{0}(4 p)\right)$ with Fourier expansion

$$
f_{d, p}(z)=q^{-d}+\sum_{0<D \equiv 0,1(\bmod 4)} A(D, d) q^{D} .
$$

An explicit construction of $f_{d, p}$ can be found in the appendix of [10] and the uniqueness of $f_{d, p}$ follows from the discussion at the end of Section 2 in [10]. The following result relates the Fourier coefficients $A(1, d)$ and $B(d)$ and shows that the traces $t^{(p)}(d)$ are Fourier coefficients of a nearly holomorphic Jacobi form of weight 2 and index $p$ (see Theorem 8 in [15] or Lemma 3.5 and Corollary 3.6 in [11]).

Theorem 3.2. Let $p$ be a prime for which $\Gamma_{0}(p)^{*}$ is of genus 0 .

(i) Let $d=4 p n-r^{2}$ for some integers $n$ and $r$. Let $A(1, d)$ be the coefficient of $q$ in $f_{d, p}$ and $B(d)$ be the coefficient of $q^{n} \zeta^{r}$ in $\phi_{p}$. Then $A(1, d)=-B(d)$.

(ii) For each natural number $d$ which is congruent to a square modulo $4 p$, let $t^{(p)}(d)$ be defined as above. We also put $t^{(p)}(-1)=-1, t^{(p)}(d)=0$ for $d<-1$. Then $t^{(p)}(d)=$ $-B(d)$.

\section{Proof of Theorem 1.3}

Proof. The proof requires the study of Hecke operators $T_{k+\frac{1}{2}, 4 p}\left(l^{2}\right)$ on the forms $g_{p}(z)$ and $f_{d, p}(z)$. Define integers $A_{l}(d)$ and $B_{l}(d)$ by

$$
A_{l}(d):=\text { the coefficient of } q \text { in } f_{d, p} \mid T_{\frac{1}{2}, 4 p}\left(l^{2}\right),
$$




$$
B_{l}(d):=\text { the coefficient of } q^{d} \text { in } g_{p}(z) \mid T_{\frac{3}{2}, 4 p}\left(l^{2}\right) .
$$

From equation (19) of [15], we have

$$
A_{l}(d)=A(1, d)+l A\left(l^{2}, d\right)
$$

Also note that we have

$$
g_{p}(z) \mid T_{\frac{3}{2}, 4 p}\left(l^{2}\right)=q^{-1}+l q^{-l^{2}}+\sum_{0<d \equiv 0,3(\bmod 4)}\left(B\left(l^{2} d\right)+\left(\frac{-d}{l}\right) B(d)+l B\left(d / l^{2}\right)\right) q^{d}
$$

and so $B_{l}(d)=B\left(l^{2} d\right)+\left(\frac{-d}{l}\right) B(d)+l B\left(d / l^{2}\right)$. Now suppose $p$ is in $\mathfrak{S}$ and $d$ is a positive integer such that $-d$ is a square modulo $4 p$ and for which an odd prime $l \neq p$ splits in $\mathbb{Q}(\sqrt{-d})$. Then $\left(\frac{-d}{l}\right)=1$. By Theorem 3.2 and the above calculations, we have

$$
\begin{aligned}
t^{(p)}\left(l^{2} d\right) & =-B\left(l^{2} d\right) \\
& =-B_{l}(d)+\left(\frac{-d}{l}\right) B(d)+l B\left(d / l^{2}\right) \\
& \equiv-B_{l}(d)+B(d) \bmod l \\
& \equiv A_{l}(d)+B(d) \bmod l \\
& \equiv A(1, d)+l A\left(l^{2}, d\right)+B(d) \bmod l \\
& \equiv-B(d)+B(d) \bmod l \\
& \equiv 0 \bmod l .
\end{aligned}
$$

Example 4.1. We now illustrate Theorem 1.3. If $p=2$ and $l=3$, then for every non-negative integer $s$, we have

$$
t^{(2)}\left(3^{2}(24 s+23)\right) \equiv 0 \bmod 3 .
$$

In particular, if we want to compute $t^{(2)}(207)$, then we are interested in $\phi_{2} \in J_{2,2}^{!}$. By Theorem 9.3 in [6] and the discussion preceding Table 8 in [15], $J_{2,2}^{!}$is the free polynomial algebra over

$$
\mathbb{C}\left[E_{4}(\tau), E_{6}(\tau), \Delta^{-1}\right] /\left(E_{4}(\tau)^{3}-E_{6}(\tau)^{2}\right)
$$

on two generators $a$ and $b$ where $\Delta=\frac{E_{4}(\tau)^{3}-E_{6}(\tau)^{2}}{1728}$. The Fourier expansions of $a$ and $b$ begin

$$
\begin{aligned}
& a=\left(\zeta-2+\zeta^{-1}\right)+\left(-2 \zeta^{2}+8 \zeta-12+8 \zeta^{-1}-2 \zeta^{-2}\right) q+\left(\zeta^{3}-12 \zeta^{2}+39 \zeta-56+\cdots\right) q^{2} \\
& +\left(8 \zeta^{3}-56 \zeta^{2}+152 \zeta-208+\cdots\right) q^{3}+\cdots \\
& \quad b=\left(\zeta+10+\zeta^{-} 1\right)+\left(10 \zeta^{2}-64 \zeta+108-64 \zeta+10 \zeta^{2}\right) q+\left(\zeta^{3}+108 \zeta^{2}-513 \zeta\right. \\
& \quad+808-\cdots) q^{2}+\left(-64 \zeta^{3}+808 \zeta^{2}-2752 \zeta+4016-\cdots\right) q^{3}+\cdots
\end{aligned}
$$

The coefficients for $a$ and $b$ can be obtained using Table 1 or the recursion formulas on page 39 of [6]. The representation of $\phi_{2}$ in terms of $a$ and $b$ is:

$$
\phi_{2}=\frac{1}{12} a\left(E_{4}(\tau) b-E_{6}(\tau) a\right) .
$$


By Theorem 3.2, we have $t^{(2)}(207)=-B(207)$. As $8 n-r^{2}=207$ has a solution $n=29$ and $r=5$, then $B(207)$ is the coefficient of $q^{29} \zeta^{5}$ which is -113643 . Thus

$$
t^{(2)}(207)=113643 \equiv 0 \bmod 3 .
$$

Remark 4.2. (1) Zagier actually defined $t^{(N)}(d)$ and proved part (ii) of Theorem 3.2 for all $N$ such that $\Gamma_{0}(N)^{*}$ is of genus 0 (see Section 8 in [15]). One might be able to prove part (i) of Theorem 3.2 in the case $N$ is squarefree. If so, then a congruence, similar to Theorem 1.3, should hold for $t^{(N)}(d), N$ squarefree. If $N$ is not squarefree, then C. Kim has kindly pointed out part (i) of Theorem 3.2 does not hold. For example, if $N=4$ and $d=7$, one can construct $f_{7,4}$ (see the appendix in [11) and compute that

$$
f_{7,4}=q^{-7}-55 q+0 q^{4}+220 q^{9}+\cdots \text {. }
$$

Thus $A(1,7)=-55$. But $B(7)=23$ (see Remark 3.1).

(2) We should note that Theorem 1.3 is an extension of the simplest case of Theorem 1 in [1. Ono and Ahlgren have also proven congruences for $t(d)$ which involve ramified or inert primes in quadratic fields. In fact, they prove that a positive proportion of primes yield congruences for $t(d)$ (see parts (2) and (3) of Theorem 1 in [1]). It would be interesting to see if such congruences hold for $t^{(p)}(d)$ or $t^{(N)}(d)$.

(3) The Monster $\mathbb{M}$ is the largest of the sporadic simple groups of order

$$
2^{46} 3^{20} 5^{9} 7^{6} 11^{2} 13^{3} 17 \cdot 19 \cdot 23 \cdot 29 \cdot 31 \cdot 41 \cdot 47 \cdot 59 \cdot 71
$$

Ogg [14] noticed that the primes dividing the order of $\mathbb{M}$ are exactly those in the set S. The monster $\mathbb{M}$ acts on a graded vector algebra $V=V_{-1} \bigoplus_{n \geq 1} V_{n}$ (see Frenkel, Lepowsky, and Meurman [7] for the construction). For any element $g \in \mathbb{M}$, let $\operatorname{Tr}\left(g \mid V_{n}\right)$ denote the trace of $g$ acting on $V_{n}$ for each $n$. Then $\operatorname{Tr}\left(g \mid V_{-1}\right)=1$ and $\operatorname{Tr}\left(g \mid V_{n}\right) \in \mathbb{Z}$ for every $n \geq 1$. The Thompson series is defined by:

$$
T_{g}(z)=q^{-1}+\sum_{n \geq 1} \operatorname{Tr}\left(g \mid V_{n}\right) q^{n} .
$$

The authors in [4] study Thompson series evaluated at imaginary quadratic arguments, i.e. "singular moduli" of Thompson series. It is possible to define a trace of singular moduli of Thompson series. A natural question is "do we have congruences for these traces?"

\section{ACKNOWLEDGMENTS}

The author would like to thank Imin Chen, Chang Heon Kim, Ken Ono, and Noriko Yui for their valuable comments.

\section{REFERENCES}

[1] S. Ahlgren, K. Ono, Arithmetic of Singular Moduli and Class Equations, to appear in Compositio Math.

[2] R. E. Borcherds, Automorphic forms on $\mathbb{O}_{s+2,2}(\mathbb{R})$ and infinite products, Invent. Math. Vol 120, (1995), 161-213.

[3] R. E. Borcherds, Automorphic forms on $\mathbb{O}_{s+2,2}(\mathbb{R})^{+}$and generalized Kac-Moody algebras, Proc. Int. Congress of Mathematicians (Zürich, 1994) (1995), 744-752.

[4] I. Chen, N. Yui, Singular values of Thompson series, in "Groups, Difference Sets and Monster" (K. T. Arau et al., Eds.), pp. 255-326, de Gruyter, Berlin, 1995.

[5] D. Cox, Primes of the Form $x^{2}+n y^{2}$, John Wiley \& Sons, Inc, New York, 1989.

[6] M. Eichler, D. Zagier, The Theory of Jacobi forms, Progress in Math. 55, Birkhäuser-Verlag, 1985.

[7] I. Frenkel, J. Leopowsky, A. Meurman, Vertex Operator Algebras and the Monster, Academic Press, New York 1988.

[8] R. Fricke, Die Elliptische Funktionen und Ihre Anwendungen, 2-ter Teil, Teubner, Leipzig 1922. 
[9] R. Fricke, Lehrbuch der Algebra III (Algebraische Zahlen), Vieweg, Braunschweig 1928.

[10] C.H. Kim, Borcherds products associated with certain Thompson series, Compositio Math. 140 (2004), 541-551.

[11] C.H. Kim, Traces of singular values and Borcherds products, preprint, 2003.

[12] N. Koblitz, Introduction to elliptic curves and modular forms, Springer-Verlag, 1984.

[13] W. Kohnen, Newforms of half-integral weight, J. reine angew. Math 333, (1982), 32-72.

[14] A. Ogg, Automorphismes de courbes modulaires, Séminaire Delange-Pisot-Poitou (Théorie des nombres) 16e année (1974/75), No. 7, 7-01-7-08.

[15] D. Zagier, Traces of singular moduli, Motives, Polylogarithms and Hodge Theory, Part I. International Press Lecture Series, editors F. Bogomolov and L. Katzarkov, International Press, Somerville (2002), 211-244.

Department of Mathematics \& Statistics, Queen's University, Kingston, Ontario, Canada K7L $3 \mathrm{~N} 6$

E-mail address: osburnr@mast.queensu.ca 